\title{
ESTATÍSTICA
}

\section{A metodologia de amostragem do Saeb”}

Marcus M. Riether

\section{Raíssa Rauter}

Palavras-chave: amostras complexas; coordenação de amostras; amostragem seqüencial de Poisson;

números aleatórios

permanentes.

\footnotetext{
* Este artigo é uma versão modificada de "Características e aspectos metodológicos da amostra para o Saeb/2001", publicado nos Anais do Marco de Aprendizagem Contínua em Avaliação, dezembro de 2001, Salvador (BA). As afirmações ou inferências contidas neste texto representam exclusivamente a visão

dos autores, não estando necessariamente respaldadas $\mathrm{e} /$ ou endossadas pelo Inep.
}

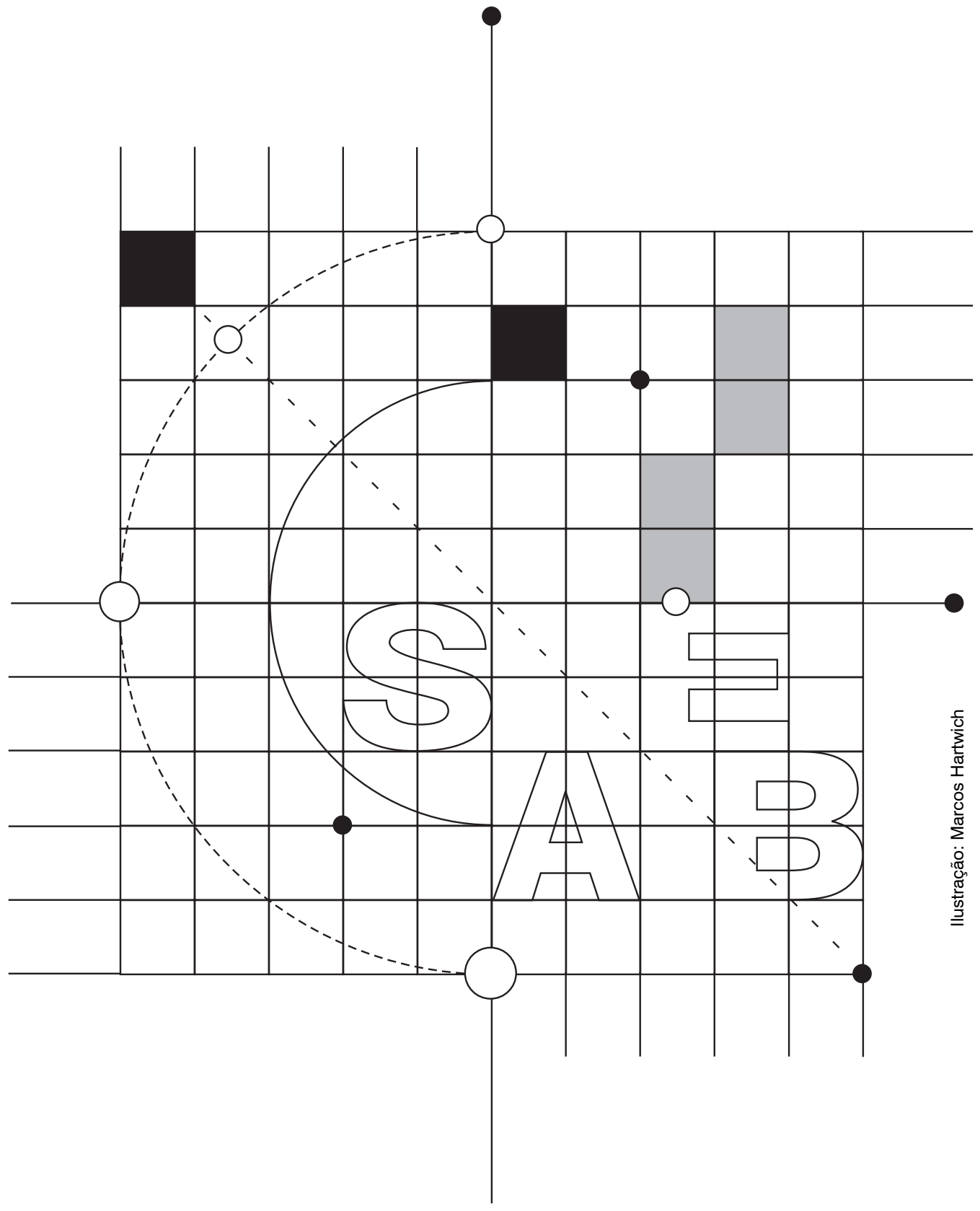




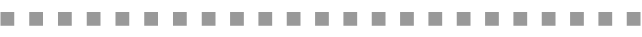

A simplificado, a metodologia de amostragem do Saeb, enfatizando particularidades relacionadas com o ciclo de 2001 e buscando esclarecer o leitor em relação a alguns dos pontos considerados mais importantes, tais como população de referência, estágios, esquemas de seleção de unidades amostrais e métodos de análise de dados de amostras complexas. Tópicos como precisão amostral, que envolvem discussões corriqueiras sobre a não-publicação de resultados individuais por escola ou a repetição de escolas que participaram do Saeb 1999, também são discutidos. Citam-se, ainda, casos de institutos de pesquisa sediados em outros países e que utilizam metodologias de amostragem semelhantes a do Saeb. Por fim, são apresentadas sugestões para o Saeb em suas futuras realizações.

\section{Introdução}

Um dos maiores e mais importantes desafios que os técnicos, direta ou indiretamente ligados ao Sistema Nacional de Avaliação da Educação Básica (Saeb), enfrentam a cada ciclo de sua realização é a produção de resultados que possam cumprir o papel a que são destinados. Entre os objetivos explícitos do Saeb está, por exemplo, a consolidação da cultura de avaliação externa do sistema de ensino regular ou o oferecimento de dados e indicadores que possibilitem maior compreensão de alguns dos fatores que influenciam o desempenho dos alunos. O próprio Instituto Nacional de Estudos e Pesquisas Educacionais (Inep) propõe que o objetivo principal do Saeb é o de "contribuir para a melhoria da qualidade da educação brasileira e para a universalização do acesso à escola, oferecendo subsídios concretos para a formulação, reformulação e o monitoramento das políticas públicas voltadas à educação básica" ou ainda "proporcionar aos agentes educacionais e à sociedade uma visão clara e concreta dos resultados dos processos de ensino e aprendizagem e das condições em que são desenvolvidos" (Instituto..., 2001).

Diante da imensurável diversidade de realidades em nosso País, cada uma delas acompanhada de suas conseqüentes necessidades, é fácil ver que os objetivos a que o Saeb se propõe são ousados em grande medida. Por isso mesmo, como é natural esperar, surge uma gama enorme de diferentes pontos de vista com que se pode olhar para o tema "avaliação educacional". Pesquisadores externos ao Inep costumam lançar críticas ao Saeb e indicar caminhos que imaginam ser mais adequados para a sua realização. É essencial lembrar, entretanto, que a realidade que ora tentamos entender é de fato muito complexa e (felizmente) nos permite enxergála de diferentes ângulos. Mas é inegável que o Inep não economiza esforços em adotar para o Saeb o uso de metodologias de ponta, muitas delas de difícil compreensão e cujo entendimento deve passar por momentos de dedicação aos diversos tópicos que envolvem a pesquisa em todas as suas fases.

Este documento procura oferecer ao leitor uma visão simplificada sobre aspectos ligados à amostra do Saeb, enfaticamente sobre o ciclo de 2001. Em particular, discute-se, procurando evitar as grandes complexidades que envolvem o assunto, que caminho seguir para produzir resultados de maneira segura e confiável, a partir dos dados brutos. É um movimento, ainda que mínimo, no sentido de fazer com que os objetivos do Saeb se aproximem mais de sua finalidade. 


\section{População de referência do Saeb 2001}

População de referência designa o conjunto total de elementos para os quais se pretende, a partir do uso de amostras, fazer inferências, buscando embasamento para a tomada de decisões, tais como o direcionamento de políticas para atendimento a grupos em desvantagem, por exemplo. No Saeb 2001, a população de referência é definida em Andrade, Silva e Bussab (2001) como

alunos da $4^{\mathrm{a}}$ ou da $8^{\underline{a}}$ séries do ensino fundamental e alunos da $3^{a}$ série do ensino médio, matriculados, em 2001, nas escolas constantes do Censo Escolar de $1999,{ }^{1}$ excetuando-se os alunos das escolas federais, rurais ou de turmas multisseriadas; para o universo da $4{ }^{a}$ série, foram mantidos ${ }^{2}$ os alunos das escolas rurais do Nordeste, MG e MS.

\section{Checagem das escolas}

A amostra do Saeb é desenhada com base em dados do Censo Escolar (amostra planejada). Por ser uma coleta transversal, o Censo não contempla (e nem poderia) variações que ocorrem entre a data de sua publicação e a de confecção das amostras do Saeb. Dessa forma, deixa de ser registrada, na época de confecção da amostra, a criação ou a extinção de escolas que tenham ocorrido nesse período, alterações cadastrais nos registros das escolas, além de que o Censo Escolar não faz a coleta discriminada das turmas e o correspondente número de alunos para cada escola. A coleta dos números de turmas, sua identificação dentro de cada escola e do número de alunos por turma é um trabalho feito em parceria com as Secretarias de Educação e se denomina "checagem das escolas", ocorrendo entre a data de confecção da amostra e a aplicação do Saeb.

No caso de escolas que tenham sido extintas, escolas que não se dispuseram a participar do Saeb (a participação não é obrigatória) ou mesmo atualizações ainda não registradas no Censo, substituições de escolas selecionadas para a amostra podem vir a ser necessárias - amostra reserva, também selecionada aleatoriamente e voltada para compensar perdas na amostra planejada, de forma a manter a precisão esperada. Na fase de planejamento da amostra, é estabelecida uma precisão mínima desejada em termos da medida de proficiência (desempenho dos alunos) ou fixado um número mínimo de elementos em cada estrato populacional. As escolas que efetivamente participam da aplicação do Saeb constituem a "amostra realizada", a partir da qual serão avaliados os porcentuais de perdas e seu impacto na qualidade dos resultados.

\section{Estratificação da população de referência e esquemas de seleção amostral}

Para que se possam produzir resultados de desempenho para as diferentes unidades da Federação e, dentro delas, resultados por dependência administrativa, por exemplo, é necessário que a população de referência seja estratificada em várias subpopulações, chamadas de estratos, e cuja característica marcante é a nãoexistência de elementos comuns entre eles (estratos independentes). A definição dos estratos é a primeira etapa na construção da amostra do Saeb, para que possa "cobrir" a maior parcela possível de áreas geográficas do País, respeitando os custos planejados e resultando em que a amostra seja representativa da população de escolas e alunos das séries pesquisadas como um todo. Além disso, a estratificação de populações excessivamente grandes $\mathrm{e}$ heterogêneas contribui para a redução da variabilidade das estimativas finais.

Em 2001, os estratos foram definidos pelas séries de interesse para a pesquisa, em seguida pela unidade da Federação, a dependência administrativa, a localização (capital ou interior) ${ }^{3}$ e o tamanho das escolas. Dentro dos estratos, o sorteio de elementos que poderão participar do Saeb ocorre em estágios distintos, tendo início com a seleção de escolas por amostragem seqüencial de Poisson e de turmas por amostragem aleatória simples.

A estratificação e o uso de probabilidades desiguais de inclusão na amostra para as escolas fazem que estimativas (médias, proporções, etc.) calculadas a partir do Saeb se comportem de maneira diferente de estimativas produzidas em amostras aleatórias simples, onde todo e qualquer aluno faz parte de um só cadastro. 
No caso do Saeb, o acesso aos alunos se dá por meio das escolas. Mesmo em relação a estas, a estratificação implica a produção de estimativas de melhor qualidade do que aquelas que seriam produzidas caso as escolas pudessem ser selecionadas com igual chance de inclusão na amostra, além de vantagens relativas aos gastos de realização do Saeb - a seleção de alunos em escolas muito pequenas e de difícil acesso, caso ocorresse, acarretaria uma elevação significativa dos custos.

\section{Implicações do uso \\ de amostragem estratificada, estágios de seleção e probabilidades desiguais de inclusão na amostra}

Uma das conseqüências advindas da forma como a amostra do Saeb é construída

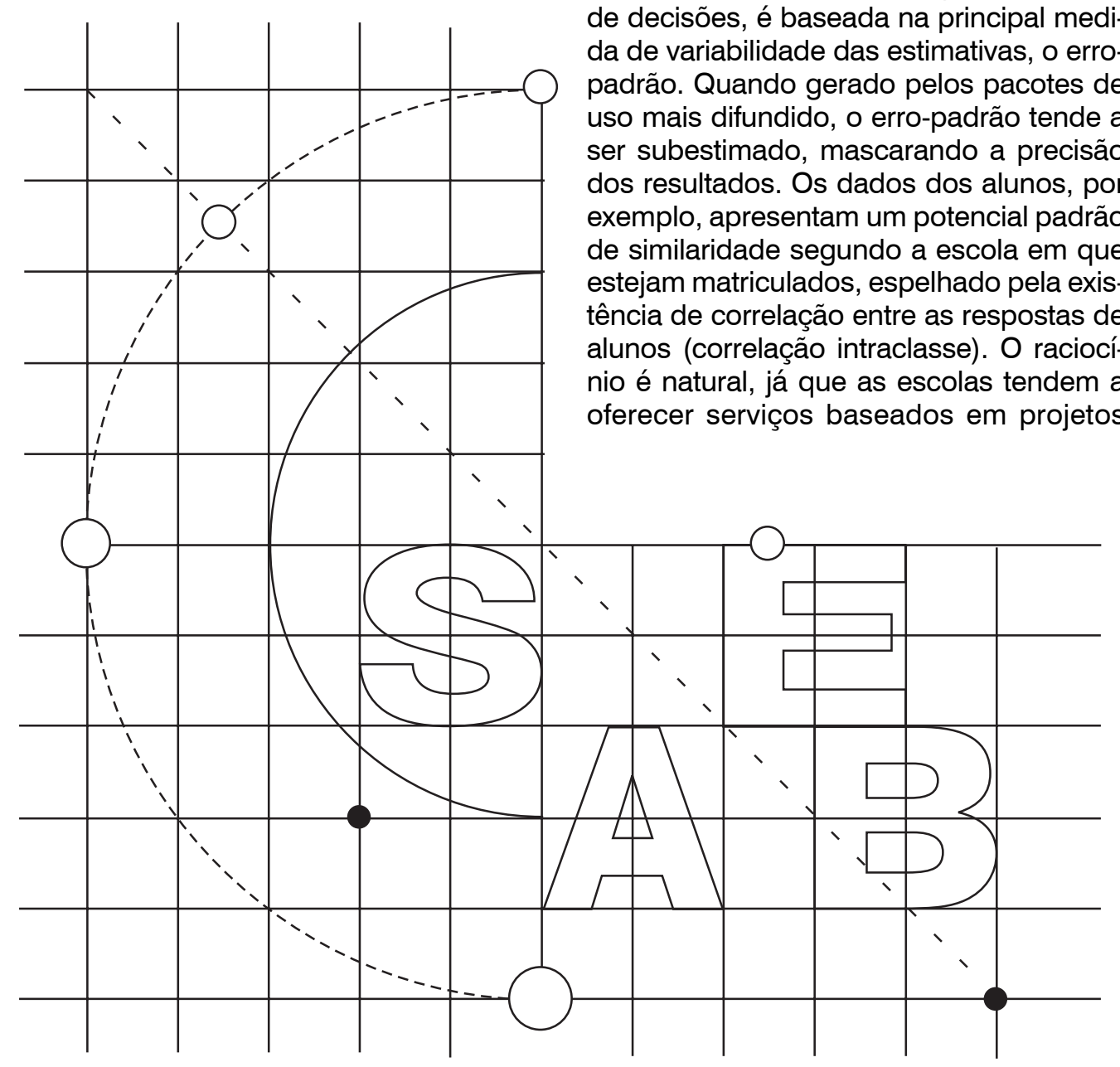

é a exigência do uso de pacotes estatísticos especializados para a obtenção de resultados válidos ou extensa programação por pessoal devidamente qualificado. Os programas mais comumente utilizados pressupõem independência das observações ou dados coletados, diferentemente do que acontece em grande parte das pesquisas em larga escala, inclusive no Saeb. Inferências que se estendam à população de referência devem ser necessariamente baseadas no uso de informações sobre o desenho amostral e dos pesos amostrais, originados nos diferentes estágios de seleção e dependentes das probabilidades de inclusão de elementos na amostra. Os pesos amostrais, acompanhados de variáveis que identifiquem a que parte (estrato) da população cada elemento pertence, devem ser usados para "calibrar" as estimativas, de forma que os resultados façam sentido para a população em sua forma original. Sem o uso dessas informações, obtêm-se estimativas pontuais viciadas (Brogan, 1998).

Além disso, a aplicação de testes estatísticos no Saeb, essenciais para a tomada de decisões, é baseada na principal medida de variabilidade das estimativas, o erropadrão. Quando gerado pelos pacotes de uso mais difundido, o erro-padrão tende a ser subestimado, mascarando a precisão dos resultados. Os dados dos alunos, por exemplo, apresentam um potencial padrão de similaridade segundo a escola em que estejam matriculados, espelhado pela existência de correlação entre as respostas de alunos (correlação intraclasse). $\mathrm{O}$ raciocínio é natural, já que as escolas tendem a oferecer serviços baseados em projetos 
pedagógicos próprios, bem como métodos de ensino, professores, entre tantos outros fatores que influenciam o dia-a-dia de seus alunos. Para o cálculo dos erros-padrão, pacotes estatísticos especializados consideram a estrutura complexa da amostra e os pesos amostrais, integrando também a correlação entre alunos e fazendo que as medidas de variabilidade carreguem consigo uma parcela devida às diferenças entre as escolas e outra devida às diferenças entre alunos dentro das escolas.

Em 1999, o Saeb passou a ter seus resultados calculados pelo Survey Data Analysis (Sudaan), um pacote de última geração utilizado para o cálculo dos erros-padrão de estimativas em amostras complexas. O Sudaan dispõe do método de linearização das séries de Taylor, adotado no Saeb, e técnicas de replicação para estimação dos erros (Shah, Barnwell, Bieler, 1997).

Estimativas simuladas no SPSS, com e sem o uso de pesos amostrais, e descontando-se o efeito do desenho amostral, quando confrontadas com aquelas corretamente geradas no Sudaan, ilustram equívocos em que se pode incorrer com uma análise inadequada dos dados. A Tabela 1 mostra as médias de desempenho em Matemática, com respectivos erros-padrão, de alunos de $4^{a}$ série, da rede estadual de ensino, por unidade da Federação, no Saeb 1999.

\section{Tabela 1 - Médias de desempenho em Matemática dos alunos de $4^{a}$ série da rede estadual de ensino, por unidade da Federação e método de cálculo das estimativas - Saeb/1999}

\begin{tabular}{|c|c|c|c|c|c|c|}
\hline \multirow{2}{*}{ UF } & \multicolumn{2}{|c|}{ SPSS sem pesos } & \multicolumn{2}{|c|}{ SPSS com pesos } & \multicolumn{2}{|c|}{ Sudaan com pesos } \\
\hline & Média & Erro & Média & Erro & Média & Erro \\
\hline Rondônia & 170,73 & 2,32 & 170,02 & 0,25 & 170,02 & 3,09 \\
\hline Acre $^{(1)}$ & - & - & & - & - & - \\
\hline Amazonas & 169,79 & 1,95 & 170,06 & 0,17 & 170,06 & 1,97 \\
\hline Roraima ${ }^{(1)}$ & - & - & - & - & - & - \\
\hline Pará & 174,74 & 2,17 & 174,30 & 0,15 & 174,30 & 3,09 \\
\hline Amapá(1) & - & - & - & - & - & - \\
\hline Tocantins & 168,26 & 2,30 & 167,29 & 0,21 & 167,29 & 3,18 \\
\hline Maranhão & 166,26 & 1,77 & 164,35 & 0,15 & 164,35 & 3,16 \\
\hline Piauí & 166,26 & 1,89 & 165,34 & 0,18 & 165,34 & 2,50 \\
\hline Ceará & 162,61 & 2,39 & 162,19 & 0,16 & 162,19 & 2,50 \\
\hline Rio G. do Norte & 162,45 & 1,81 & 161,85 & 0,17 & 161,85 & 4,24 \\
\hline Paraíba & 171,35 & 1,96 & 172,15 & 0,19 & 172,15 & 2,54 \\
\hline Pernambuco & 161,12 & 2,18 & 159,63 & 0,13 & 159,63 & 2,73 \\
\hline Alagoas & 168,28 & 2,10 & 168,93 & 0,27 & 168,93 & 3,66 \\
\hline Sergipe & 164,83 & 1,95 & 167,61 & 0,23 & 167,61 & 3,48 \\
\hline Bahia & 165,00 & 2,17 & 165,66 & 0,10 & 165,66 & 2,26 \\
\hline Minas Gerais & 182,71 & 3,12 & 187,46 & 0,10 & 187,46 & 9,88 \\
\hline Espírito Santo & 179,03 & 2,36 & 179,23 & 0,21 & 179,23 & 3,19 \\
\hline Rio de Janeiro & 175,31 & 2,63 & 173,52 & 0,14 & 173,52 & 4,49 \\
\hline São Paulo & 185,10 & 2,64 & 184,60 & 0,06 & 184,60 & 3,60 \\
\hline Paraná & 191,35 & 2,66 & 190,37 & 0,24 & 190,37 & 4,40 \\
\hline Santa Catarina & 190,14 & 2,11 & 190,11 & 0,15 & 190,11 & 2,01 \\
\hline Rio G. do Sul & 188,66 & 2,50 & 188,40 & 0,12 & 188,40 & 2,97 \\
\hline Mato G. do Sul & 177,67 & 2,29 & 179,92 & 0,24 & 179,92 & 3,81 \\
\hline Mato Grosso & 174,62 & 2,40 & 173,87 & 0,19 & 173,87 & 2,86 \\
\hline Goiás & 184,19 & 2,40 & 182,01 & 0,14 & 182,01 & 3,72 \\
\hline Distrito Federal & 176,58 & 2,31 & 176,24 & 0,19 & 176,24 & 1,38 \\
\hline
\end{tabular}


Nota-se que, quando os pesos e o desenho amostral são desprezados, obtêm-se médias incorretas. Quando considerados apenas os pesos, ainda no SPSS, as estimativas pontuais são idênticas às geradas no Sudaan, mas os erros-padrão se apresentam bastante subestimados. Testes de comparação entre médias, nesse caso, facilmente acusariam diferenças significativas falsas entre alguns Estados.
Os Gráficos 1 e 2 apresentam os intervalos de confiança $(95 \%)$ gerados respectivamente pelo SPSS, sem o uso de pesos amostrais, e pelo Sudaan, considerando os pesos e o desenho amostral. Das 146 relações significativas apontadas pelo SPSS na apuração de diferenças entre médias das unidades da Federação, 50 (34\%) estariam equivocadas, considerando-se as análises geradas pelo Sudaan.

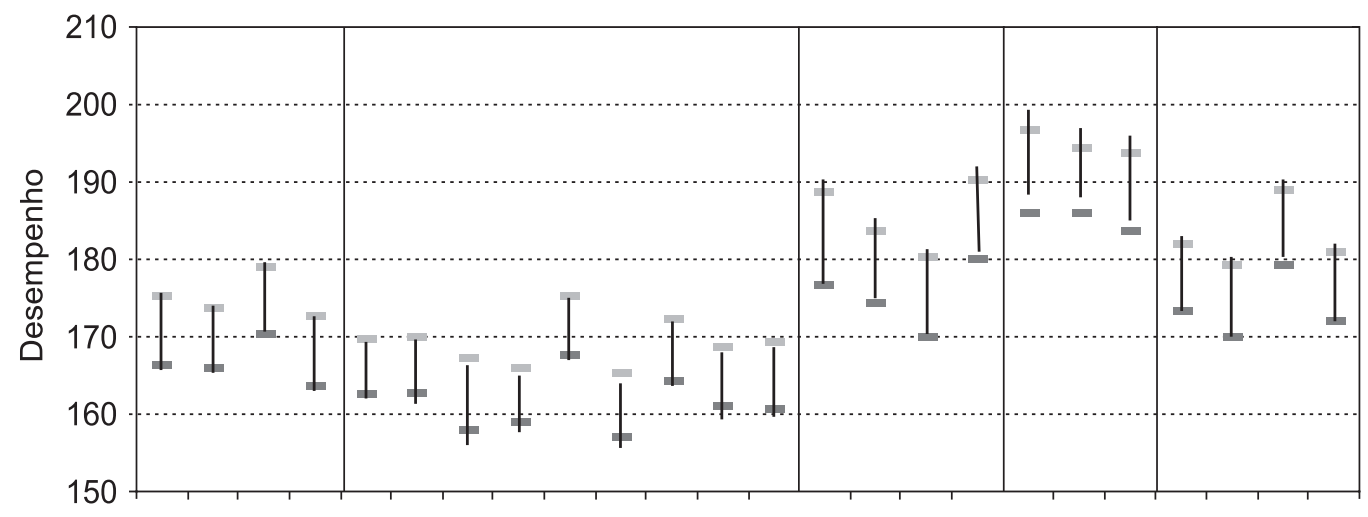

RO AM PA TO MA PI CE RN PB PE AL SE BA MG ES RJ SP PR SC RS MS MT GO DF

UF

$$
\text { - Limite Inferior - Limite Superior }
$$

Gráfico 1 - Intervalo de confiança do desempenho dos alunos de 4a série da rede estadual, em Matemática - Saeb/1999 - Cálculo pelo SPSS, sem uso de pesos amostrais

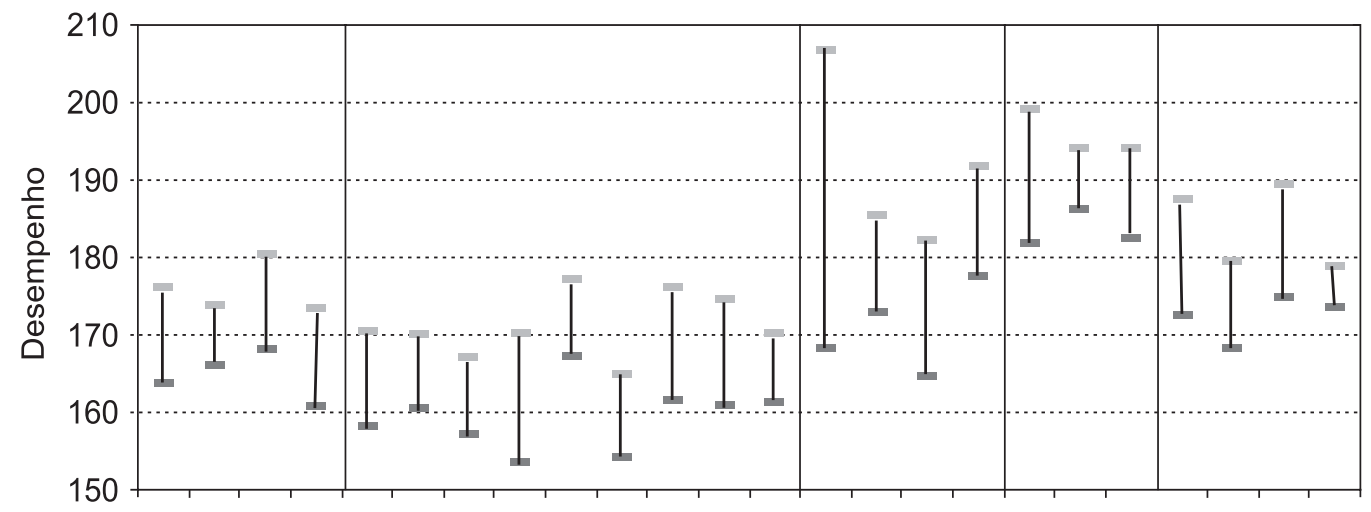

RO AM PA TO MA PI CE RN PB PE AL SE BA MG ES RJ SP PR SC RS MS MT GO DF

UF

- Limite Inferior $\quad$ Limite Superior

Gráfico 2 - Intervalo de confiança do desempenho de alunos de 4a série da rede estadual, em Matemática - Saeb/1999 - Cálculo pelo Sudaan, com pesos e considerando o desenho amostral 


\section{Tamanho da amostra versus precisão dos resultados}

Os diferentes tamanhos de amostras apresentados na Tabela 2 obedecem, em primeiro lugar, à disponibilidade de recursos para realização do Saeb a cada ciclo. A precisão amostral é definida a partir da divisão da população em estratos que contenham um número mínimo de alunos e também de escolas, juntamente com a programação de um número desejado e igual de alunos selecionados em cada estrato. Para o Saeb 2001, busca-se a mesma precisão amostral para todos os estratos, perseguida com base em padrões obtidos em 1999.

Tabela 2 - Evolução da amostra do Saeb - 1995 a 2001

\begin{tabular}{|c|c|c|c|c|}
\hline \multirow{2}{*}{ Participantes } & \multicolumn{4}{|c|}{ Ano de realização } \\
\hline & 1995 & 1997 & 1999 & 2001 \\
\hline Escolas & 2.839 & 1.933 & 6.890 & 6.935 \\
\hline Diretores & 2.839 & 1.933 & 6.890 & 6.831 \\
\hline Professores & 4.971 & 13.267 & 43.793 & $22.042^{*}$ \\
\hline Alunos & 90.499 & 167.196 & 279.764 & 287.719 \\
\hline $\begin{array}{l}\text { Séries } \\
\text { avaliadas }\end{array}$ & $\begin{array}{c}4^{a} \text { a } 8^{a} E F \text { e } \\
2^{a} \text { a } 3^{a} E M\end{array}$ & $\begin{array}{c}4^{a} \text { a } 8^{a} E F ~ e \\
3^{a} E M\end{array}$ & $\begin{array}{c}4^{a} \text { a } 8^{a} E F \text { e } \\
3^{a} E M\end{array}$ & $\begin{array}{c}4^{a} \text { a } 8^{a} E F \text { e } \\
3^{a} E M\end{array}$ \\
\hline \multirow[t]{2}{*}{$\begin{array}{l}\text { Disciplinas } \\
\text { avaliadas }\end{array}$} & \multirow[t]{2}{*}{$\begin{array}{l}\text { Matemática } \\
\text { Língua } \\
\text { Portuguesa }\end{array}$} & \multirow[t]{2}{*}{$\begin{array}{c}\text { Matemática } \\
\text { Língua } \\
\text { Portuguesa } \\
\text { Ciências }\end{array}$} & $\begin{array}{c}\text { Matemática } \\
\text { Língua } \\
\text { Portuguesa } \\
\text { Geografia } \\
\text { História } \\
\text { Ciências }\end{array}$ & \multirow[t]{2}{*}{$\begin{array}{c}\text { Matemática } \\
\text { Língua } \\
\text { Portuguesa }\end{array}$} \\
\hline & & & $\begin{array}{ll}\text { na } 3^{\mathrm{a}} & \text { Biologia } \\
\text { série }= & \text { Física } \\
\text { Ciências } & \text { Química }\end{array}$ & \\
\hline
\end{tabular}

Fonte: MEC/Inep/Daeb.

* A estimativa de número de professores a serem pesquisados baseia-se em que serão avaliados um professor para cada turma de $4^{\mathrm{a}}$ série e dois professores (um por disciplina) para cada turma de $8^{\mathrm{a}}$ e $3^{\mathrm{a}}$ séries.

Segundo a regra de que a precisão amostral dos resultados do Saeb está ligada à existência de quantidades mínimas de unidades avaliadas, deve-se ressaltar que os resultados não são válidos para grupos pequenos de alunos, como seria, por exemplo, a média de desempenho para uma única escola. Afirmações a partir desse tipo de estimativa devem ser evitadas, já que tais resultados, quando vistos individualmente, podem ser (e provavelmente são) equivocados e certamente não oferecem qualquer parcela de representatividade quando confrontadas com a população de referência do Saeb. Este é um resultado estatístico e, portanto, absolutamente desvinculado do uso que se espera fazer dos dados da pesquisa.

\section{Coordenação das amostras de escolas}

As amostras dos Saeb realizados em 1999 e 2001 tiveram, como ponto em comum, a seleção de escolas por amostragem seqüencial de Poisson. A Amostragem de Poisson tem a vantagem de ser um procedimento de fácil implementação e é usada para a seleção aleatória de elementos que apresentam probabilidades desiguais de inclusão numa amostra. Deve-se ressaltar, contudo, que todos os elementos pertencentes à população de referência ou, em outras palavras, as escolas registradas no Censo Escolar contidas nos estratos pré-definidos, têm probabilidades reais de inclusão na amostra e, portanto, de participarem do Saeb. 
Porém, uma das principais características da amostragem de Poisson é que o tamanho da amostra gerado por esse esquema é aleatório, e uma das conseqüências advindas desse fato é que as estimativas produzidas sob amostragem de Poisson carregam consigo também uma parcela de variabilidade proveniente do tamanho da amostra. A forma encontrada para se conseguir a variabilidade mínima possível das estimativas consiste, por exemplo, na escolha de medidas adequadas de tamanho das escolas, que representam, no caso do Saeb, as unidades primárias amostrais (elementos sorteados em primeiro lugar). A medida de tamanho das escolas utilizada no Saeb 2001 foi o número de turmas das séries pesquisadas em cada grupo de estratos correspondente.

A principal diferença entre os esquemas seqüencial e não-seqüencial está em que o primeiro é uma adaptação do esquema de Poisson que leva à confecção de amostras de tamanho fixo (Ohlsson, 1998), implicando o maior controle sobre os custos da pesquisa.

A amostra do Saeb 2001 contou, ainda, com a associação de números aleatórios permanentes às escolas no Censo, levando à coordenação positiva entre as amostras dos Saeb 1999 e 2001 (repetição de escolas nas duas pesquisas - ver Tabela 3). A coordenação de amostras tem como finalidade oferecer ao pesquisador a possibilidade de maximizar ou de minimizar a repetição, na amostra a ser sorteada, de elementos selecionados em amostras de ciclos anteriores ou de elementos em amostras paralelas. No caso do Saeb, a repetição de um certo número de escolas e o uso de variáveis comuns entre ciclos reforça a precisão das estimativas de mudança ${ }^{4}$ (Ohlsson, 1995).

Tabela 3 - Sobreposição de amostras do Saeb ao longo dos anos - 1995 a 2001

\begin{tabular}{|l|r|r|r|r|}
\hline & \multicolumn{4}{|c|}{ Ano de realização } \\
\cline { 2 - 5 } & 1995 & 1997 & $1999^{*}$ & 2001 \\
\hline $\begin{array}{l}\text { Número de escolas na amostra } \\
\text { Número de escolas que se repetem em reação }\end{array}$ & 2.839 & 1.933 & 6.890 & 6.935 \\
$\begin{array}{l}\text { à aplicação imediatamente anterior } \\
\text { Porcentual de sobreposição }\end{array}$ &.. & $\ldots$ & 696 & 3.490 \\
\hline & & $\ldots$ & $10,1 \%$ & $49,6 \%$ \\
\hline
\end{tabular}

A técnica de Números Aleatórios Permanentes também é aplicada no Saeb em busca da máxima sobreposição possível de escolas que tenham turmas de mais de uma das séries avaliadas, de forma a promover a máxima redução dos custos de acesso às unidades a serem visitadas (Andrade, Silva, Bussab, 2001).

Na prática, a técnica consiste em associar a cada uma das escolas constantes do Censo Escolar, dentro das séries de interesse, um número sorteado aleatoriamente, classificando as escolas do Censo em ordem crescente. A partir da população assim classificada, escolas são sorteadas em zonas de amostragem com espalhamento geográfico conseguido pela ordenação das escolas pelo Código de Endereçamento Postal (CEP) e na quantidade desejada para se atingir o número de alunos necessários para se obter a esperada precisão amostral. A adoção desse procedimento aumenta as possibilidades do Saeb como instrumento de diagnóstico, por exemplo, de fatores que impactam o desempenho dos alunos, permitindo, com a realização de alguns novos ciclos, que estudos de natureza longitudinal venham a ser produzidos.

Cabe ressalvar, apenas, que algum cuidado deve ser tomado em relação a que aspectos poderiam ser avaliados em estudos longitudinais. A participação no Saeb se dá, primeiramente, no que se refere a variáveis próprias da escola, em seguida variáveis do diretor (ou diretora, naturalmente), caso este tenha sido mantido no cargo por mais de um ciclo de avaliação, seguidas pelas variáveis de professor. Escolas que se repetem podem não

\footnotetext{
Em inglês, usa-se o termo change para se referir à mudança ou variação que um dado fenômeno sofre ao longo do tempo.
} 


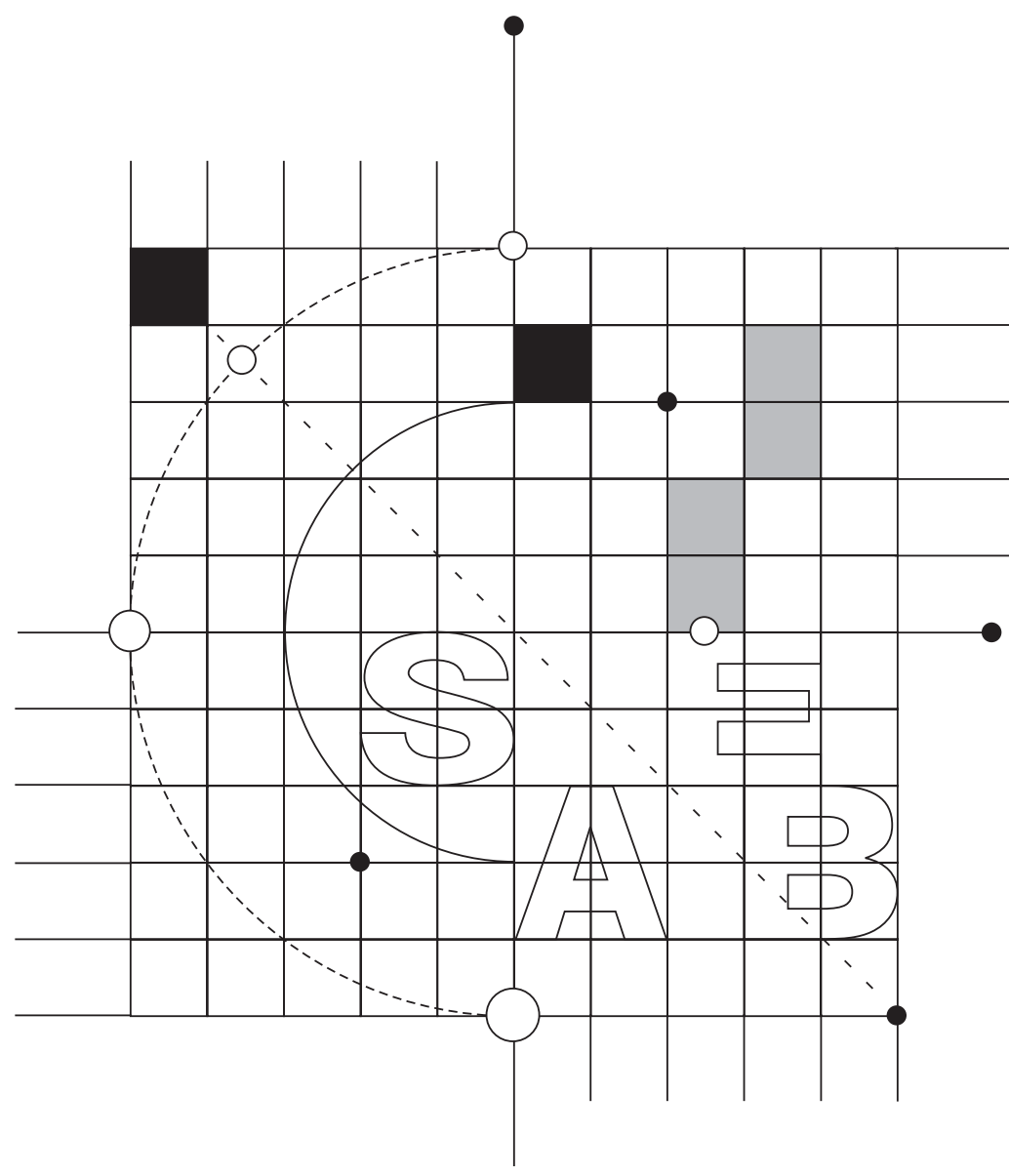

ter o mesmo diretor na avaliação seguinte e muito menos os mesmos professores, sobretudo porque as turmas também participam do Saeb via sorteio. Quanto aos alunos, quando se pensa nas séries avaliadas, tem-se que um aluno, digamos, da $4^{a}$ série, somente poderia participar de uma nova avaliação quando atingisse a 8 $^{a}$ série (o Saeb é bianual), caso continuasse na mesma escola, sem ter repetido o ano, e estivesse novamente matriculado em uma turma sorteada. Assim, variáveis próprias do aluno só muito raramente viriam a se repetir partindo do mesmo sujeito. Talvez o maior ganho que se possa extrair desse tipo de estudo seja no tocante a métodos de ensino ou aspectos ligados à gestão e à sua relação com o desempenho dos alunos.

\section{Outras pesquisas que utilizam metodologia semelhante a do Saeb}

A metodologia de coordenação de amostras, hoje adotada no Saeb, é prática comum já há muitos anos em institutos de pesquisa em diversos países, tais como Suécia, Austrália, Nova Zelândia e França, por exemplo, geralmente associados a pesquisas na área de estabelecimentos comerciais, mas também é prática corrente em várias pesquisas sociais geridas pelo Instituto Brasileiro de Geografia e Estatística (IBGE).

\section{Saeb e as avaliações estaduais}

Uma outra possibilidade que o uso de números aleatórios permanentes abre é a de coordenação positiva de amostras entre o Saeb e avaliações estaduais realizadas por amostragem. Na verdade, o tema surge como uma questão a ser discutida em foros mais amplos, envolvendo profissionais de diversas origens. A sobreposição de um certo número de escolas entre pesquisas paralelas, tipo Saeb e avaliações estaduais, aparece, por exemplo, como opção para a redução dos custos totais, que poderiam ser repartidos entre financiadores em níveis federal e estadual, à parte implicações políticas disso decorrentes, inclusive no que se refere aos interesses subjacentes à prática de realização de avaliações externas de cada uma das esferas de governo. Nesse caso, o Saeb e os Estados poderiam fazer uso da mesma base de dados gerada pela realização de apenas uma prova, podendo ou não promover a equalização de resultados também de forma conjunta. Com isso, também no sentido da redução de custos, os Estados poderiam, opcionalmente, sujeitar apenas uma subamostra adicional de escolas a terem todas as suas turmas avaliadas, esquivandose dos gastos com avaliações censitárias e mesmo assim aumentando o leque de opções das análises de dados. Por exemplo, a aplicação de modelos multinível aos dados poderia vir a apresentar, a despeito de questionamentos advindos de alguns pesquisadores da área, resultados surpreendentes. Por outro lado, a coordenação negativa de amostras (inexistência de elementos comuns) também aparece como uma possibilidade e poderia garantir que alunos que fizessem provas do Saeb não participariam de avaliações estaduais, retirando o ônus dos estudantes em ter que responder a mais de uma avaliação externa, provavelmente um incômodo para a maioria.

\section{Refinamento das relações entre 0 avaliado e 0 avaliador externo}

Voltando, por fim, aos objetivos do Saeb, parece útil lembrar que o bem maior que se pode produzir com a prática de 
avaliações educacionais é dar ao jovem condições para que possa assumir, com segurança e desenvoltura, compromissos em seu dia-a-dia. Disso faz parte o estreitamento das relações entre quem avalia e quem é avaliado.

Nesse sentido, o relacionamento entre o Inep e as Secretarias de Educação aparece, certamente, como uma via de duas mãos. Tanto importa ao Inep aprofundar o conhecimento do dia-a-dia nas escolas, estas muito mais próximas das Secretarias que do Ministério, levando a que os "fazedores" de políticas públicas possam entender com mais propriedade as necessidades daqueles para quem as políticas são primordialmente dirigidas, quanto importa aos gestores de escolas entenderem e acompanharem os processos de avaliação em nível nacional, colhendo informações sobre tendências nacionais ou regionais, dificilmente captadas quando se cruzam resultados produzidos por métodos de avaliação não-padronizados e em escopos muito menos amplos.

\section{Referências bibliográficas}

ANDRADE, D. F.; SILVA, P. L. N.; BUSSAB, W. O. Plano Amostral para o Saeb 2001: Final Draft. Brasília, 2001.

BROGAN, D. J. Pitfalls of using standard statistical software packages for sample survey data. In: ARMITAGE, P.; COLTON, T. (Ed.). Encyclopedia of Biostatistics. New York: John Wiley and Sons, 1998.

BUSSAB, W. O. et al. Plano Amostral Saeb/99: Memo no 2 - Definição do Plano Amostral. Brasília, 1999.

ERNST, L. R. Maximizing and minimizing overlap when selecting a large number of units per stratum simultaneously for two designs. Journal of Official Statistics, Sweden, v. 14, n. 3, p. 297-314, 1998.

INSTITUTO NACIONAL DE ESTUDOS E PESQUISAS EDUCACIONAIS. Saeb 2001 Novas Perspectivas. Brasília: Inep, 2001.

LINDBLOM, A. SAMU - The System for Sample Co-ordination from the Business Register at Statistics Sweden. Statistics Sweden, S-701 89 Örebro, Sweden, 1999. Disponível em <http://europa.eu.int/en/comm/eurostat/research/conferences/etk-99/ papers/lindblom.pdf > Acesso em novembro de 2001.

OHLSSON, E. Coordination of samples using permanent random numbers. In: COX, B. G. et al. Business Survey Methods. New York: John Wiley, 1995. p. 153-170.

. Sequential poisson sampling. Journal of Official Statistics, Sweden, v. 14, n. 2, p. 149-162, 1998.

SHAH, B. V.; BARNWELL, B. G.; BIELER, G. S. SUDAAN User's Manual, Release 7.5. Research Triangle Park, NC, USA: Research Triangle Institute, 1997.

Recebido em 25 de fevereiro de 2002.

Marcus M. Riether, bacharel em Estatística pela Universidade de Brasília (UnB), é consultor do Serviço Social da Indústria (Sesi). marcusriether@hotmail.com

Raíssa Rauter, mestranda em Psicologia Social e do Trabalho pela UnB, é consultora da Diretoria de Avaliação da Educação Básica (Daeb) do Instituto Nacional de Estudos e Pesquisas Educacionais (Inep). raissa@inep.gov.br 


\section{Abstract}

This paper presents a simplified version of the Saeb sampling methodology, emphasizing particularities of its cycle of 2001, and aiming at making clear to the reader some of its main points, such as target population, stages, sampling schemes and data analyses methods applied to complex samples. Topics like precision of estimates, that involve customary discussions about the avoidance in publishing schools' individual results or the sample overlap with the Saeb 1999 are also discussed. Cases of international research institutes that make use of sampling methodologies similar to that of Saeb are also mentioned. To finish, we present suggestions to Saeb in its future realizations.

Keywords: complex samples; sample overlap; sequential Poisson sampling; permanent random numbers. 